\title{
Estudos in silico ADME para uma série de inibidores da enzima conversora da angiotensina (ECA)
}

\author{
Yuri Rayel Fernandes de Sousa ${ }^{1}$, Joaquim S. da Costa Júnior ${ }^{1}$, \\ Danielle da Costa Silva ${ }^{1}$
}

${ }^{1}$ Curso de Química, Laboratório de Química Orgânica dos Produtos Naturais, Instituto
Federal do Piauí (IFPI) - Campus Teresina Central, Teresina-PI, Brasil
yurirayel101@gmail.com, jquimjr@ifpi.edu.br, dcsdanielle@gmail.com

\begin{abstract}
Arterial hypertension is considered the main factor of morbimortality in the world, and it is necessary to explore other forms of treatment that accessible more and that allow an effective blockade of the related pathophysiological mechanism. Thus, the main objective of the present work is to evaluate the pharmacokinetic parameters of in silico metabolization for molecules derived from triazolones and bis-triazolones that inhibit the angiotensin-converting enzyme (ACE). The parameters were analyzed using the PreADMET program. The results obtained are unprecedented and show that the selected compounds have considerable properties for inhibiting CYP enzymes.
\end{abstract}

Resumo. A hipertensão arterial é tida como principal fator de morbimortalidade no mundo, por isso é necessário explorar outras formas de tratamento mais acessíveis e que possibilitem um efetivo bloqueio do mecanismo fisiopatológico envolvido. Assim, o presente trabalho tem como objetivo principal avaliar os parâmetros farmacocinéticos de metabolização in silico para moléculas derivadas de triazolonas e bis-triazolonas inibidoras da enzima conversora da angiotensina (ECA). Os parâmetros foram analisados por meio da utilização do programa PreADMET. Os resultados obtidos são inéditos e evidenciam que os compostos selecionados possuem propriedades consideráveis para inibição das enzimas $C Y P$.

\section{Introdução}

A hipertensão arterial (HA) faz parte do grupo de doenças crônicas não-transmissíveis (DCNTs) e, também, é classificada como doença circulatória, pois resulta de diversas alterações nos sistemas que atuam no controle da pressão arterial [Krieger; Irigiyen and Krieger 1999]. A HA é um problema de Saúde Pública e, entre as diversas doenças do sistema cardiovascular, a patologia citada é conhecida como o principal fator de morbimortalidade humana no mundo [Carvalho et al. 2003]. Assim, faz-se necessário explorar outras formas de tratamentos mais acessíveis e que possibilitem um efetivo bloqueio do mecanismo fisiopatológico da HA, independente da sua via de formação.

Uma área que permite entender os mecanismos de controle da pressão arterial é a Química Medicinal (QM). Por ser um campo de alta complexidade, é preciso realizar a interação com outras áreas do conhecimento, devido sua característica multidisciplinar, abrangendo: Bioquímica, Farmacologia, Informática, Química Computacional, Química Orgânica, Biologia Molecular e Estrutural, devido os aspectos 
relacionados à descoberta, direcionando a busca na melhoria de diagnósticos e tratamentos, assim como a elaboração de substâncias bioativas de interesse terapêutico notáveis, bem como sua interação com os alvos moleculares no organismo, na obtenção de fármacos mais potentes e com menos efeitos colaterais [Carvalho et al. 2003].

Por outro lado, ao utilizar métodos de Inteligência Artificial (IA), por meio do aprendizado de máquinas (Machine Learning - ML), com o intuito de planejar fármacos mais específicos e aplicações bem definidas, a QM têm proporcionado a construção de modelos preditivos robustos e bem aprimorados devido as ferramentas tecnológicas utilizadas, com redução do tempo e dos custos de produção de fármacos. Desta forma, este trabalho tem como objetivo principal avaliar os parâmetros farmacocinéticos de metabolização in silico para moléculas derivadas de triazolonas e bis-triazolonas inibidoras da ECA, corroborando assim, com o planejamento de fármacos antihipertensivos e um melhor entendimento dos mecanismos fisiopatológicos envolvidos.

\section{Revisão da Literatura}

O sistema renina-angiotensina (SRA) é considerado como uma cascata hormonal [Silva et al. 2014], por determinar a hemodinâmica e homeostase cardiovascular [Oigman and Neves 2000] e desempenhar um papel importante inter-relacionado o mecanismo de controle do volume, pressão e composição eletrolítica do sangue [Lima 1999].

A proteína hepática precursora do SRA é o angiotensinogênio (AGT), que é clivado pela renina, liberada pelo rim, devido ao alto nível de sal no organismo. Em seguida, no sangue, a renina origina a angiotensina I (AI). Esta é, então, convertida pela ECA a angiotensina II (AII) [Cat and Touyz 2011]. A AI passa pela circulação pulmonar, onde, acontece a reação de hidrólise pela ECA no C-terminal His-Leu, convertendo a AI para AII. A AII é um agente vasoconstrictor importante, responsável pela reabsorção de sódio e síntese de aldosterona, além de interagir com dois subtipos de receptores distintos: $\mathrm{AT}_{1}$ e $\mathrm{AT}_{2}$ [Garrido and Griendling 2009].

A ECA tem um papel duplo na vasculatura sanguínea na medida em que promove a produção de AII, estimulando também a degradação da bradicinina [Cat and Touyz 2011], daí a importância de realizar estudos in silico ADME de compostos com atividade anti-hipertensiva que atuem na ECA, baseado no planejamento de fármacos.

O planejamento de fármacos auxiliado por computador ("Computer-Assisted Drug Design"- CADD) investiga as interações químicas de um ligante com o seu alvo molecular e explora os fatores estruturais relacionados ao efeito biológico. Assim, a elucidação dos mecanismos envolvidos, podem ser compreendidas pela agregação de conhecimentos fundamentais presentes na área de QM [Carvalho et al. 2003].

As pesquisas envolvendo CADD estão direcionadas no desenvolvimento e/ou descoberta de novos agentes terapêuticos, com o uso de métodos de IA, tais como: modelagem molecular, propriedades farmacocinéticas e toxicológicas (absorção, digestão, metabolismo, excreção e toxicologia (ADMET)), bem como de propriedades moleculares, por meio de estudos envolvendo métodos de mecânica quântica.

A aplicação envolvendo CADD têm despertado grande interesse dos cientistas, universidades, indústrias farmacêuticas e empresas de biotecnologia, devido à redução de tempo e dos altos custos envolvidos para a produção de fármacos, pois pode viabilizar a otimização das propriedades farmacocinéticas e farmacodinâmicas [Salum 
and Andricopulo 2009]. E, sabe-se que a disponibilidade de programas computacionais de química e os bancos de dados em rede são, atualmente, ferramentas fundamentais para a descoberta e planejamento de fármacos [Carvalho et al. 2003].

\section{Materiais e Métodos}

\subsection{Conjunto de Dados}

Após um levantamento criterioso na literatura científica, selecionou-se 5 moléculas, que foram sintetizadas em laboratório e que apresentavam atividade inibitória para a ECA [Ben Salah et al. 2018]. As estruturas químicas de todas as moléculas usadas neste estudo, foram numeradas de 1 a 5 e são descritas na Tabela 1.

Tabela 1. Conjunto de dados [Ben Salah et al. 2018]

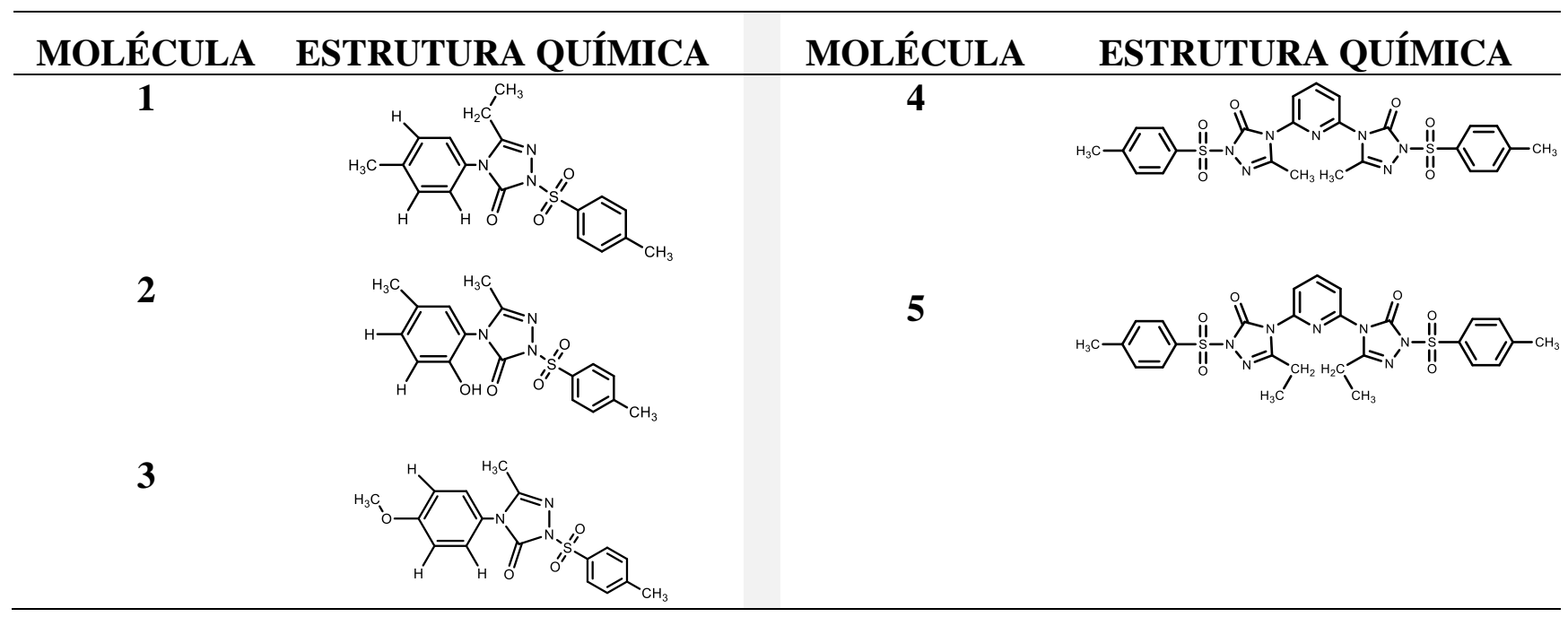

\subsection{Base de Dados para a Predição das Propriedades Farmacocinéticas}

Para a realização do estudo in silico de predição das propriedades farmacocinéticas do conjunto de compostos bioativos selecionados, utilizou-se o programa baseado na web PreADMET (disponível em: https://preadmet.bmdrc.kr/).

O software PreADMET reside inteiramente em um servidor Web. O aplicativo é escrito principalmente em PHP, que por sua vez, usa um conjunto de programas C que implementam muitas das suas funcionalidades. Este consiste em quatro partes principais: (i) Cálculo do descritor molecular; (ii) Predição da semelhança com drogas; (iii) Predição de toxicidade e (iv) Predição de ADME (disponível em: https://preadmet.bmdrc.kr/). Para realização do estudo apresentado neste trabalho, utilizou-se a funcionalidade Predição de ADME. A fim de construir os modelos preditivos robustos no estudo das propriedades farmacocinéticas in silico, a aproximação funcional genética foi usada para selecionar descritores relevantes de todos os descritores 2D calculados pelo módulo Topomol, na parte de Cálculo do descritor molecular, seguido por rede neural resiliente de retropropagação (Rprop) [Riedmiller and Braun 1993] para desenvolver um modelo não-linear de sucesso. 


\section{Resultados e Discussão}

\subsection{Caracterização do Conjunto de Dados}

Os compostos selecionados foram sintetizados e testados in vitro por Ben Salah e colaboradores [2018] e são potentes inibidores da ECA (iECA) [Ben Salah et al. 2018]. Até o presente momento, não foram encontrados estudos in silico ADME publicados na literatura para os compostos selecionados.

Os compostos selecionados apresentam ação: anti-hipertensiva, antimicrobiana, antitumoral, anti-inflamatória e anticâncer [Ben Salah et al. 2018]. Em vista disso, estudos recentes de docagem molecular, interação proteína-ligante, realizados por [Ben Salah et al. 2018], demonstram que ligantes com anéis de triazolona possuem ótimos resultados de iECA, por meio das interações presentes na bolsa catalítica da enzima.

De acordo com [Gao et al. 2019], o grupo 1,2,4-triazol influencia na lipofilicidade, polaridade e capacidade de ligação de hidrogênio da molécula, melhorando as propriedades físico-químicas e possuem uma série de propriedades farmacológicas, além de reduzir a toxicidade e melhorar os perfis farmacocinéticos.

\subsection{Análise dos Parâmetros Farmacocinéticos (ADME) in silico}

Utilizou-se os seguintes programas: software freeware ChemSketch ACDLabs (versão 10.04, 2008), para o desenho das estruturas químicas; software PreADMET (versão 2.0, Copyright( 2005), para realizar as análises in silico dos estudos ADME para o conjunto de dados. Nesta primeira etapa, foi realizado estudo dos parâmetros metabólicos. A Tabela 2 mostra os resultados obtidos para os parâmetros analisados.

Tabela 2. Resultados obtidos para o parâmetro de metabolização

MOLÉCULAS

\begin{tabular}{cccccc}
\hline PARÂMETROS & $\mathbf{1}$ & $\mathbf{2}$ & $\mathbf{3}$ & $\mathbf{4}$ & $\mathbf{5}$ \\
\hline CYP2C19 inibição & Não & Não & Não & Não & Não \\
CYP2C9 inibição & Não & Inibidor & Não & Inibidor & Inibidor \\
CYP2D6 inibição & Não & Não & Não & Não & Não \\
CYP2D6 substrato & Não & Não & Não & Não & Não \\
CYP3A4 inibição & Não & Não & Não & Inibidor & Inibidor \\
CYP3A4 substrato & Substrato & Não & Substrato & Substrato & Substrato \\
SK (logD) & 3,964 & 3,188 & 2,802 & 3,930 & 5,194 \\
SK $(\operatorname{logP})$ & 3,964 & 3,188 & 2,802 & 3,930 & 5,194 \\
SK $(\operatorname{logS})$ & $-2,389$ & $-1,851$ & $-1,885$ & $-2,968$ & $-3,767$ \\
SK $\operatorname{logS}$ em água pura & $-5,405$ & $-4,673$ & $-4,489$ & $-7,786$ & $-8,936$ \\
\hline
\end{tabular}


Os resultados mostrados na Tabela 2 evidenciam que nenhum composto inibe a CYP2C19. Já as moléculas 2, 4 e 5 apresentam inibição da CYP2C9. O conjunto de dados apresentou efeito substrato e não inibitório para a CYP2D6. Os compostos 4 e 5 inibem a CYP3A4, e, apenas o composto 2 não atua como substrato da CYP3A4.

O sistema oxidativo Citocromo P450 (CYP450) é relevante nos estudos de metabolismo em ADME, por codificar enzimas, sendo as principais: CYP2C9, CYP2C19, CYP2D6 e CYP3A4, pois possibilitam a metabolização de 90\% das drogas. Essas são expressas em maior proporção no fígado, mas estão também presentes no intestino delgado. A CYP3A, i. e., metaboliza mais de $50 \%$ dos fármacos e possibilita a metabolização de antidepressivos, neurolépticos e diazepínicos [Silvado 2008].

A magnitude do efeito de inibição ou indução pode mudar conforme a fração do metabolismo da droga que é realizada pela enzima que é induzida (aumenta atividade enzimática) ou inibida (reduz atividade enzimática). A inclusão de indutores da CYP450 no regime terapêutico do paciente pode ter como principal consequência clínica a redução da efetividade de um dos fármacos. Contudo, as consequências da inibição incluem aumento da toxicidade do medicamento afetado pela interação, ou redução da efetividade, quando o medicamento é um pró-fármaco e depende da atividade de enzimas CYP450, para ativar a CYP2C19 [Silvado 2008].

É importante descrever que moléculas bioativas que inibem apenas uma CYP podem induzir a redução do número de fármacos que interagem farmacocineticamente, assim como compostos que inibem duas ou mais enzimas CYP, em especial, a CYP3A4 e CYP2C9, podem interferir no metabolismo de uma enorme gama de fármacos, o que de certa forma acarreta no aumento da sua toxicidade no organismo. Vale destacar que compostos não inibidores e não indutores de CYP são tidos como ideais, por não interferirem no metabolismo de outras drogas [Dolabela, M. F. et al 2018].

Os descritores: coeficientes de partição (LogP), solubilidade (LogS) e distribuição $(\log \mathrm{D})$ estão voltados para as análises físico-químicas dos compostos. $\mathrm{O}$ LogP, $i$. e., está relacionado com a capacidade de um composto se particionar em solventes, água, meio aquoso, tampão ou também fluidos sanguíneos e, é um parâmetro frequentemente usado em estudos in silico, como medida quantitativa da lipofilicidade de moléculas bioativas [Barreiro and Fraga 2014]. Já o LogS, representa a concentração do composto em mol/L de uma solução aquosa saturada em equilíbrio. E, por fim, o LogD, destaca a lipofilicidade de um composto em um dado $\mathrm{pH}$, além de ser uma função de contribuição ao $\operatorname{LogP}$ [Barreiro and Fraga 2014].

Os resultados dos parâmetros para o $\log \mathrm{P}, \log \mathrm{D}$ e $\operatorname{LogS}$ mostram que o composto 5 obteve maiores valores de partição e distribuição, enquanto o composto 2 tem maior valor para $\operatorname{LogS}$ em solução tampão com $\mathrm{pH} 7,4$, que representa o pH sanguíneo. Por fim, o coeficiente $\log S$ em água pura foi maior para o composto 2.

\section{Conclusão e Trabalhos em Andamento}

Os resultados obtidos neste trabalho evidenciam que os parâmetros farmacocinéticos de metabolização in silico para moléculas derivadas de triazolonas e bis-triazolonas inibidoras da enzima conversora da angiotensina (ECA), são inéditos. Observou-se que grande parte dos compostos estudados possuem propriedades consideráveis para inibição das enzimas CYP. 
Entretanto, uma investigação mais aprofundada precisa ser realizada, para uma melhor avaliação acerca dos compostos estudados. Assim, além da análise do parâmetro já realizado, outros estão sendo avaliados, $i$. e., absorção, distribuição, excreção e toxicidade para um melhor entendimento da bioavaliabilidade oral.

\section{Agradecimentos}

Os autores agradecem a Fundação de Amparo à Pesquisa do Estado do Piauí (FAPEPI), Conselho Nacional de Desenvolvimento Científico e Tecnológico (CNPq) e ao Instituto Federal do Piauí (IFPI).

\section{Referências}

Barreiro, E. J. and Fraga, C. A. M. (2014). “Química Medicinal: As Bases Moleculares da Ação dos Fármacos". Artmed: Porto Alegre, $3^{\mathrm{a}}$ edição, p. 47-50.

Ben, S. B. et al. (2018). "Design, synthesis of novel Triazolones and bis-Triazolones derivatives under ultrasound irradiation and evaluation as potent angiotensin converting enzyme (ACE) inhibitors". Bioorganic Chemistry, vol. 76, p. 147-153.

Carvalho, I. et al. (2003). "Introdução a modelagem molecular de fármacos no curso experimental de Química Farmacêutica”, Quím. Nova, vol. 26 (3), pag. 428-438.

Cat, A. N. D. and Touyz, R. M. (2011). "A new look at the renin-angiotensin systemFocusing on the vascular system". Peptides, v. 32, n. 10, p. 2141-2150.

Dolabela, M. F. et al. (2018). "Estudo in silico das atividades de triterpenos e iridoides isolados de Himatanthus articulatus (Vahl) Woodson". Fitos, vol. 12, p. 227-242.

Gao, F. et al. (2019). "Antibacterial activity study of 1,2,4-triazole derivatives". European Journal of Medicinal Chemistry, vol. 173, p. 274-281.

Garrido, A. M. and Griendling, K. K. (2009). "NADPH oxidases and angiotensin II receptor signaling”. Mol. Cell. Endocrinol. vol. 302, p. 148-158.

Krieger, E. M.; Irigiyen, M. C. and Krieger, J. E. (1999). "Fisiopatologia da hipertensão". RSCESP, v. 9, n. 1, p. 01-07.

Lima, D. P. (1999). "Synthesis of angiotensin-converting enzyme (ACE) inhibitors: an important class of antihypertensive drugs". Quím. Nova, vol. 22, n. 3, p. 375-381.

Oigman, W. and Neves, M. F. T. (2000). "Sistema renina-angiotensina e hipertrofia ventricular esquerda". Rev Bras Hipertens, vol. 07, n. 03, p. 261-267.

Riedmiller, M. and Braun, H. (1993). "A direct adaptive method for faster backpropagation learning: The Rprop algorithm". IEEE International Conference on Neural Networks (ICNN), vol. 01, p. 586-591.

Salum, L. B. and Andricopulo, A. D. (2009). "Frgment-based QSAR: perspectives in drug design". Mol. Divers, vol. 13, p. 277-285.

Silva, D. C. et al. (2014). "Understanding electrostatic and steric requirements related to hypertensive action of AT1 antagonists using molecular modeling techniques". Journal of Molecular Modeling, vol. 20, n. 7, p. 01-14.

Silvado, C. (2008). "Farmacogenética e antiepilépticos (farmacologia das drogas antiepilépticas: da teoria à prática)". J. epilepsy clin. Neurophysiol, vol. 14, p. 51-56. 